\title{
V. Electric discharge in a transverse magnetic field
}

\author{
Prof. D.N. Mallik B.A. Sc.D. F.R.S.E. \& A.B. Das M.Sc.
}

To cite this article: Prof. D.N. Mallik B.A. Sc.D. F.R.S.E. \& A.B. Das M.Sc. (1916) V. Electric discharge in a transverse magnetic field, Philosophical Magazine Series 6, 32:187, 50-65, DOI: $10.1080 / 14786441608635543$

To link to this article: http://dx.doi.org/10.1080/14786441608635543

$$
\text { 曲 Published online: } 08 \text { Apr } 2009 .
$$

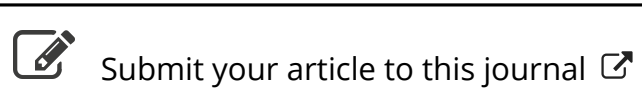

Џلl Article views: 2

Q View related articles 두 
V. Electric Discharge in a Transverse Magnetic Field. By Prof. D. N. MaLLIK, B.A., Sc.D., F.R.S.E., and A. B. Das, M.Sc.*

\section{[Plate II.]}

1. $\mathrm{N}$ a paper in the Philosophical Magazine, Oct. 1908, one of us (D. N. M.) considered the behaviour of an electric discharge in a tube of De La Rive's pattern, under gradually decreasing pressure. It was there shown that, starting with a pressure inside the tube equal to that of the atmosphere, the discharge is at first in the nature of a shower of sparks, filling the whole tube; that these various streams combine into a single band or single thick stream, when the pressure is sufficiently low ; that only when the discharge is of this kind is there rotation under the action of a magnetic field, as in the usual De La Rive apparatus. It was also noted that, as the pressure is further reduced, the band is gradually changed into a shower again and the rotation ceases.

2. In a subsequent paper (Oct. 1912) a theoretical explanation of the various phenomena was attempted, and it was shown that they are connected with the fact that the relative number of positive and negative ions present in the tube varied at these different stages.

3. The object of the present paper is to discuss, in detail, the behaviour of the discharge when the rotatory stage is passed. It results both from theory and experiment that the phenomena are dependent on (1) the voltage of the primary of the induction-coil, (2) the nature of the coil, (3) the difference of potential between the electrodes of the discharge-tube, (4) their distance, (5) and (6) the pressure and the nature of the gas or vapour in the tube, and (7) the nature of the electrodes.

It has been our object to investigate the manner in which the phenomena are severally dependent on them.

4. That the variation in the character of the discharge depends on the induction-coil used to produce the discharge and the distance between the electrodes in the dischargetube is clearly seen from the photographs (Pl. II. figs. 1, $2,3,4)$.

* Communicated by the Authors. Papor based on brief notes communicated to the second and the third All-India Science Congresses (1915 and 1916). 
Fig. 1. "Band" discharge which rotates when the electromagnet is excited. (Pressure 55 millimetres.)

Fig. 2. "Glow" discharge at pressure 2 millimetres. (The induction-coil (A) used in these cases gives a spark-length in atmospheric air of 29 millimetres.)

Figs. $3 \& 4$ are photograph of the discharge at the same pressures as in 1, 2 respectively, with the inductioncoil (B) giving a spark-length in atmospheric air of 13.4 millimetres. The discharge-tube (No. 1) is the same in both cases. In the latter group, no band discharge appears, and consequently the rotatory effect is absent throughout the entire range. With a smaller tube (No. 2), however, and the inductioncoil (B) the discharge shows the various types referred to on page 50 .

5 . It is a priori evident that there must be a definite relation between pressure in the tube, the voltage of the induction-coil, and the length of rotatory discharge. What the nature of this relation should be would appear from the following considerations.

If $n$ is the number of corpuscles per unit length (along $x$ ) per unit area of cross-section of a discharge, then the equation of continuity (J. J. Thomson, "Conduction of Electricity through Gases') is

$$
\frac{\partial n}{\partial t}+\frac{\partial(n u)}{\partial t}=\frac{n u}{\lambda}\left[f\left(\frac{\mathrm{X} e}{p}\right)-\beta\right]
$$

where $u$ is the velocity of a corpusele;

$\lambda=$ mean free path ;

$f$ is a function, which determines the number of collisions resulting in ionization, of the mean kinetic energy $\mathrm{X} e \lambda$ of an ion given to it by the electrical field of intensity $\mathrm{X}$;

$\beta$ is the fraction of collisions resulting in recombination ;

$p=$ pressure ;

$e=$ charge on an ion $(+$ and -$)$.

Now, for steady rotation both $\frac{d n}{d t}$ and $\frac{\partial n u}{\partial x}$ must vanish, and we must have

$$
\begin{gathered}
f\left(\frac{\mathrm{X} e}{p}\right)-\beta=0 \text { or } f\left(\frac{\mathrm{X} e}{p}\right)-\beta=0 . \\
\therefore \frac{\mathrm{X} e}{p}=\beta^{\prime}, \text { say. }
\end{gathered}
$$

E 2 
If we assume $\beta^{\prime}$ to be of the form $\frac{a}{p}+b$, the $\mathrm{X}, p$ curve is a straight line, and if $a$ is small, $\frac{\mathrm{X}}{p}=$ const.

As $\mathrm{X}$ and probably also $f$ and $\beta$ depend on the distance between the electrodes and the voliage of the inductioncoil, there uust be an exact relation between pressure \&c. determining the condition under which the discharge becomes rotatory. This exact quantitative relation is under investigation.

6. Starting now from the stage at which the discharge is rotatory, we reach, when the pressure is gradually reduced, a stage at which the character of the discharge changes as in figs. $5,6,7$.

7. Comparing the figs. 5 and 6 , which give the initial and the final stages of a discharge at the same pressure, we observe that the illumination is very slight when the discharge is first passed, but that after a time it becomes much more marked. The effect is evidently due to the fact that when the discharge is first passed there is only slight ionization, but that, as the discharge is continued, ionization increases and the consequent illumination. 7 corresponds to a lower pressure than 6 .

8. When this stage is reached, the ring-end of the discharge is found to spread over a finite length of the ring electrode, instead of being confined practically to one point in it.

9. As the pressure is further reduced, there is a further spreading out as in fig. 7 .

10. If at these stages the electromagnet is excited, there is dispersal of the streams constituting the discharge, which is, at the same time, twisted about the Faraday dark space on either side of it.

Figs. 8 and 9 show the effect of the magnetic field on fig. 6 , which is a photograph of the discharge before the electromagnet is excited.

Similarly figs. 10 and 11 correspond to fig. 7 of the nonmagnetic field. In 8 and 11 the disk is the cathode, and in 9 and 10 the ring is the cathode.

11. If we admit that the twist is due to an angular displacement of ions about the axis of the electromagnet, this behaviour of the Faraday dark space [10] must be due to the fact that it is a region practically devoid of ions.

12. Fig. $12 a$, curve I. gives the amount of twist for different current strengths in the coil of the electromagnet. It is thus easily seen that since the magnetic force is 
proportional to current strength, within the limits of the actual experiment, the twist is also proportional to the intensity of

Fig. 12 a. Curve I.

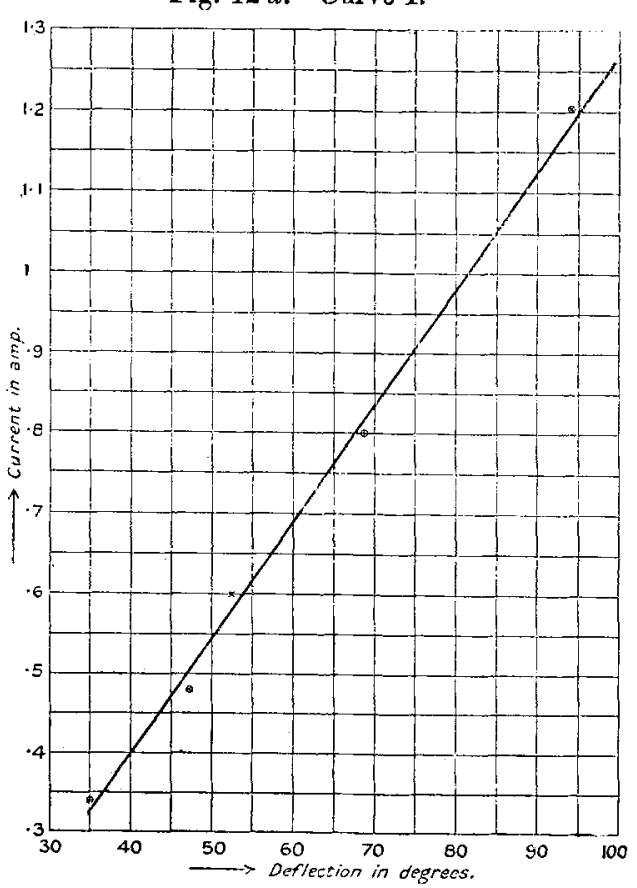

[Nature of the discharge.-When the induction-coil is started, nearly the whole space is dark, and then a bright band with a dark space near the cathode gradually appears.]

the magnetic force. Fig. $12 b$, eurve II. gives the amount of i wist for different current strengths at a lower pressure. It is seen that the corresponding twists are greater in this case than those at a higher pressure.

13. An approximate theory of the magnetic action of the excited magnetic field on these discharges can be worked out as follows:-

Using cylindrical coordinates, $z, \rho, \theta$ (where $z$ is measured from the Faraday dark space [11], the equations of motion of an ion may be written, if $m$ is its mass,

$$
\begin{aligned}
& m \ddot{z}+\mathrm{A} \dot{z}=\mathrm{Z}_{e}+\mathrm{H}_{e} \dot{\rho} \dot{\theta}, \quad \text {. . . . . . (1) } \\
& m\left(\ddot{\rho}-\rho \dot{\theta}^{2}\right)+\mathrm{A} \dot{\rho}=\mathrm{R}, \quad . \quad . \quad . \quad . \quad . \quad . \\
& m \frac{1}{\rho} \frac{d}{d t}\left(\rho^{2} \dot{\theta}\right)+\mathrm{A} \rho \dot{\theta}+\mathrm{B} \theta=\mathrm{H} e \dot{z}, \quad . \quad .
\end{aligned}
$$

where $A=$ coeff. of viscosity, $B$ a coeff. to be determined, 
54 Prof. D. N. Mallik and Mr. A. B. Das on Eleetric

$\mathrm{Z}=$ electric force in the direction of $z$ and $\mathrm{R}$ in the direction of $\rho$, while $\mathrm{H}=$ magnetic force, which we know is mainly in the direction of $\rho$ (Phil. Mag. Jan. 1908).

Fig. $12 b$. Curve II.

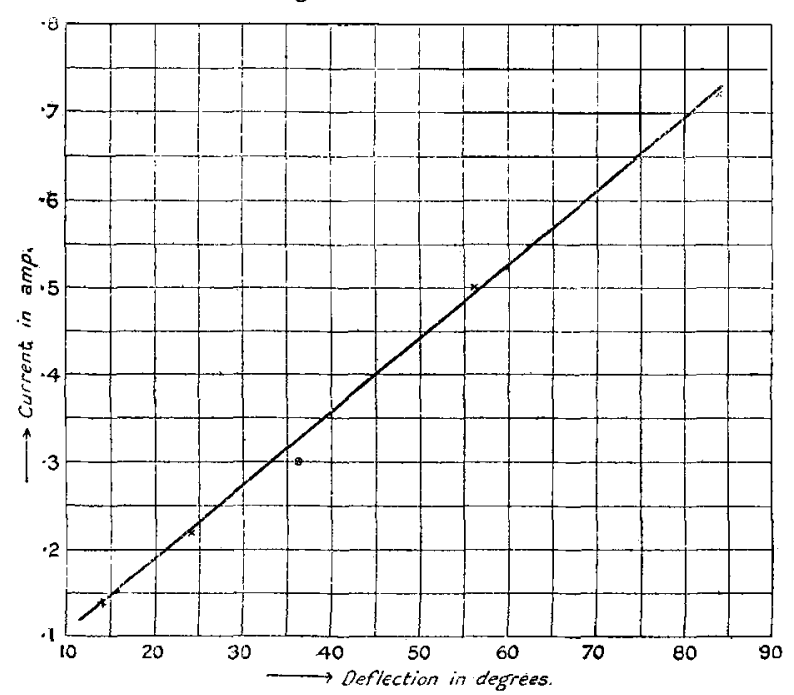

[Nature of the discharge.-Showery, with very fine striæ. It takes a certain time for the striæ and illumination to develop after the induction-coil is started.]

Now, considering the equation (3) (to which, alone, we shall confine ourselves), if we have $N$ negative and $n$ positive ions per unit length, in any stream (màsses $m_{1}$ and $m_{2}$ respectively), we have, taking moments about the axis and summing up,

$$
\begin{aligned}
\int\left(m_{1} \mathrm{~N}+m_{2} n\right) d s \frac{d}{d t}\left(\rho^{2} \dot{\theta}\right)+\int\left(\mathrm{A}_{1} \mathrm{~N}+\mathrm{A}_{2} n\right) \rho^{2} d s \dot{\theta} \\
+\int \mathrm{B} \rho(\mathrm{N}+n) d s \theta=\int \mathrm{H} \rho e(\mathrm{~N}+n) d s \dot{z} .
\end{aligned}
$$

as the equation of motion of any stream of discharge.

Now we may assume, as in Phil. Mag. Oct. 1912, the action between two streams of lengths $d s, d s^{\prime}$ to be a repulsion

$$
=\frac{e^{2} d s d s^{\prime}}{\mathrm{K} r^{2}} \cdot\left[(\mathrm{N}-n)^{2}-\frac{\left(n q+\mathrm{N} q^{\prime}\right)^{2}}{3 \mathrm{~V}^{2}}\right]+\frac{\pi \rho}{r^{3}}\left(n a^{2} q-\mathrm{N} b^{2} q^{\prime}\right)^{2},
$$

where $q, q^{\prime}$ are the velocities of positive and negative ions, $a, b$ their radii (assumed sphorical), $r$ the distance between $d s, d s^{\prime}, \mathrm{K}$ the S.I.C. and V the velocity of light. 
The third term of (4) will then be of the form

$$
\mathrm{C} f(\alpha)(n-\mathrm{N})^{2} \theta \text {, }
$$

the other terms (depending on the velocities) being neglected. Here $\mathrm{C}$ is a constant depending on the form of the various streams of discharge, and $\alpha$ the angular coordinate defining the position of the stream, whose equation of motion is given by (4), provided $n$ and $\mathrm{N}$ are constant throughout the discharge, observing that in this case, alone, $\theta$ will be the same for all points. In any case, if $n=\mathrm{N}$, the equation of motion is of the form

$$
\mathrm{I} \ddot{\theta}+\mu \dot{\theta}=\int \mathrm{H}_{\rho i} i d s
$$

$=$ the couple acting on the discharge due to the magnetic action of the electromagnet,

where

$$
\begin{aligned}
& \mathbf{I}=\int\left(m_{1}+m_{2}\right) n d s \rho^{2}, \\
& \dot{\rho}=0, \text { in the steady state. }
\end{aligned}
$$

sines

But this couple $=\frac{3}{2} \mathbf{M} i$, where $M$ is the total magnetic strength of induced magnetism (Phil. Mag. Oct. 1908).

Therefore we bave

$$
\mathrm{I} \ddot{\theta}+\mu \dot{\theta}=\frac{3}{2} \mathrm{M} i,
$$

where

$$
\mu=\left(\mathrm{A}_{1}+\mathrm{A}_{2}\right) n \int \rho^{2} d s .
$$

This is the same equation as was obtained in a previous paper by identifying the discharge (which is in the form of fig. 1) with an electric current.

14. If the number of positive particles is small in comparison with that of negative particles, the number of the latter will not necessarily be constant throughout any stream of discharge. In this case, putting $n=0$ and considering the motion of a small element of a discharge, we have, when the steady stage is reached,

$$
\mathrm{B} \theta=\mathrm{H} e \dot{z} \quad \text { or } \quad \theta=\frac{\mathrm{H} e \dot{z}}{\mathrm{~B}}, \quad . \quad . \quad \text {. }
$$

where $B$ is a function of $\rho, \alpha$, defining the position of the element of the discharge considered. This completely explains the twists described in para. 10.

Comparing figs. 8 and 11 with figs. 9 and 10, one might be led to suppose that the twist is independent nf the direction 
of the discharge. This is not really the case, as is seen from the annexed diagram.

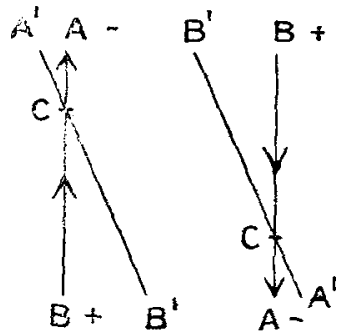

$\mathbf{A}$ is the cathode, $\mathbf{B}$ is the anode, $\mathrm{C}$ the dark space.

Curve II. is also easily explained by the fact that at a lower pressure $\dot{z}$ is greater than at a higher pressure.

15. The equation (5) shows that the angular displacement of a corpuscle is proportional to $\mathrm{H}$ and is dependent on its distance from the asis as well as on its angular position. The latter explains the dispersal of the rays [10]. The equation (3) also shows that there will be a time-factor; that is, the final effect will be reached only gradually. Experiment bears this out also.

16. When the pressure is further reduced, the discharge becomes striatory (PI. II. fig. 13). On the introduction of the magnetic field, the Faraday dark space shortens in length, the number of striæe increases, the distance between consecutive strix decreises, and they also bulge out (fig. 14).

The effect of increasing the magnetic field is to increase the number of strixe and further shorten the length of the Faraday dark space, as well as that between consecutive stria.

17. On a still further reduction of pressure, all the striæ disappear and the negative glow extends from the eathodo to nearly the whole of the tube (fig. 15).

At-this stage, the discharge is luminous at first, but the luminosity decreases as the discharge continues to pass, in consequence, evidently, of a gradual recombination of ions.

18. When the electromagnet is excited, the striæ make their appearance, and the tube is illuminated with a reddish light (figs. $16 \& 17$ ). Fig. 17 shows the effect of increased magnetic field. 
19. When the pressure is very low (about $\frac{1}{1000}$ of a millimetre) the negative glow reaches the sides of the vessel and the whole tube becomes phosphorescent, owing evidently to the impact of corpuscles on the sides of the tube.

On the excitation of the maynetic field, the phosphorescent effect becomes concentrated around the cathode, while the anode is surrounded by a red luminosity, the line of demarcation between the two regions being clearly marked.

20. A characteristic variation of the potential difference with pressure during these changes is (as was noted in a previous paper) the most marked feature of these experiments. In order to make a more detailed study of this variation than was attempted previously - through all ranges of pressureit was necessary, in the first place, to standardize the E.M.F. available with the induction-coils $A$ and $B$. For this, we observed the spark-distance between two brass spheres, each of radius $3 \mathrm{~cm}$, , when the voltage of the primary of the induction-coil was gradually changed, the spring of the interrupter being kept at a constant tension. The result is plotted in curve III., fig. 18, in which ordinates represent spark-lengths in miliimetres and the abscissa the voltage of the primaries of the induction-coil $\mathrm{A}$.

Fig. 18. Curve III.

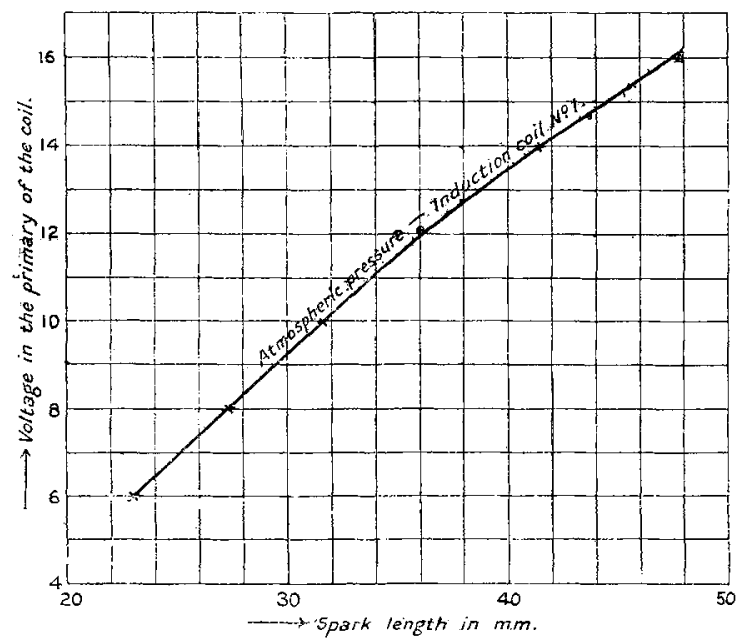

21. Comparing these with the result given in page 461 of J. J. Thomson's 'Discharge of Electricity through Gases,' 
and similarly plotted in curve IV., fig. 19, we come to the conclusion that the voltage of the secondary of an induction coil, under the conditions of these experiments, is fairly accurately measured by the distane $\theta$ of the minimum sparkgap, across which it forces a discharge and is practically proportional to this distance.

Fig. 19. Curve IV.

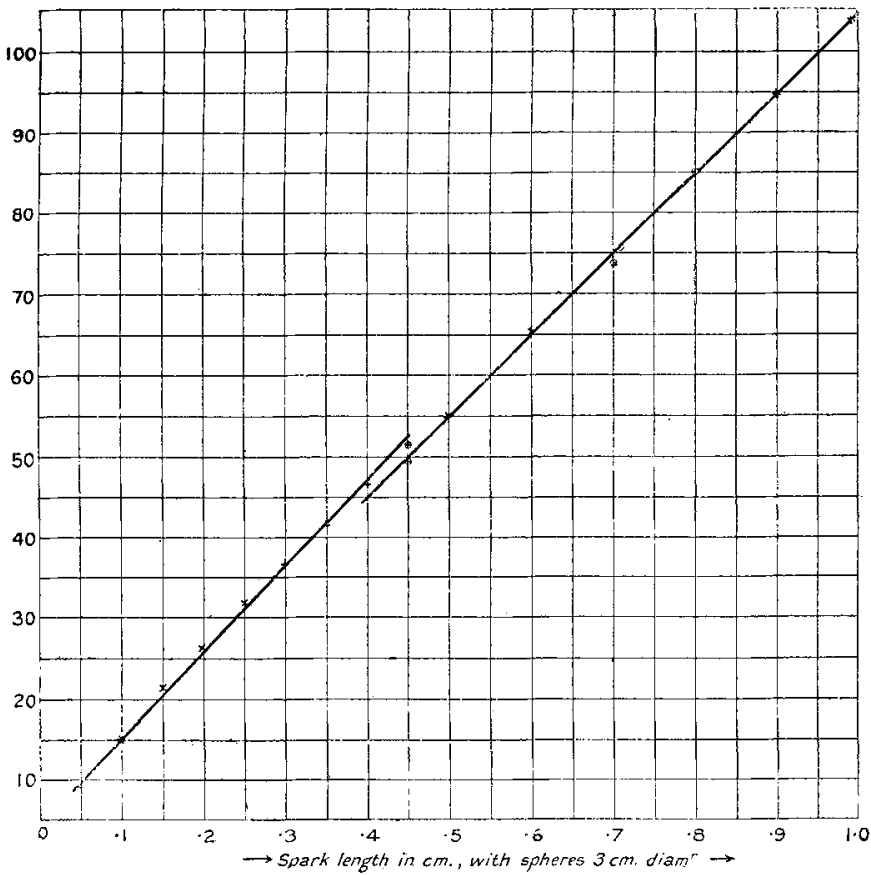

[Data from Baillie's results-J. J. Thomson's ' Conduction of Electricity through Gases,'2nd ed. page 461.]

It follows also that the potential difference between the electrodes of the discharge-tube is measurable in terms of the minimum spark-gap with which it is parallel. In this way, the potential difference between the electrodes of the discharge-tube at different pressures of the contained air has been determined.

22. Fig. 20, curve V. represents changes in the P.D. with pressure, when the disk is the cathode, the sparklength of the induction-coil in this case being $13.4 \mathrm{~mm}$. in atmospheric air. 
Curve VI. represents the relation between these quantities under similar circumstances with the ring as cathode.

These curves correspond to the series of discharges typically represented by figs. 3 and 4 . The pressures to which these figures actually relate are, however, comparatively low.

Throughout this series of changes of pressure, the rotatory stage is absent.

Fig. 20. Curres V. \& VI.

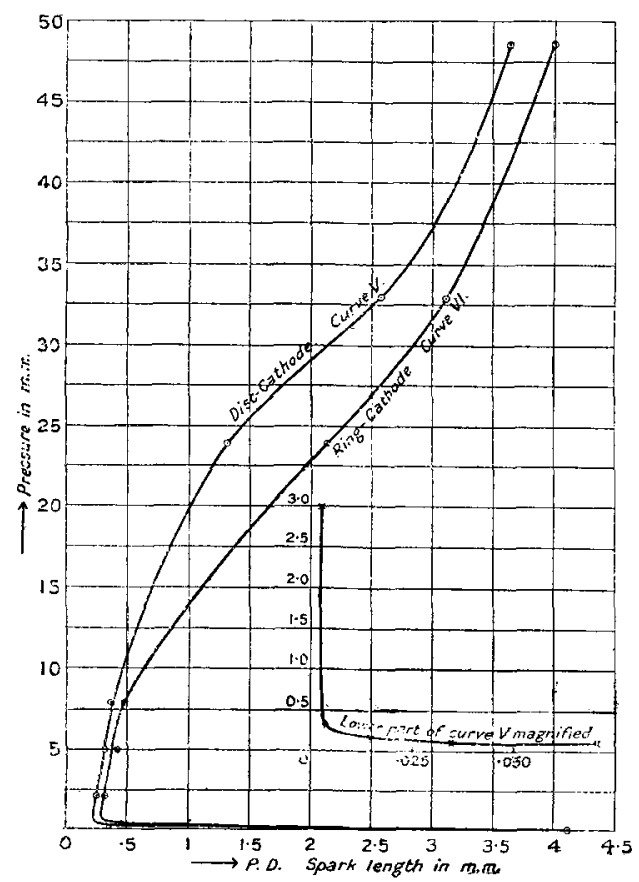

23. In fig. 21, curve VII. represents changes in P.D. with pressure between the electrodes of the same tube with disk as the cathode, the spark-length of the induction-coil in this case being $29 \mathrm{~mm}$. in atmospheric air. It corresponds to the series of discharges typified by figs. 1 and 2 .

It will be observed that the latter includes a stage at which discharge has, during a certain range of pressure, the appearance of a band, and that there is rotation, only when this state is reached, under the influence of a radial magnetic force. 
The straight part A, B of curve VII. corresponds to the rotatory stage of which fig. 1 is the type. The bent part $B, C$ of curve VII. corresponds to the range of pressure over which the discharge undergoes only a twist [10] under the magnetic force.

Fig. 21. Curve VII,

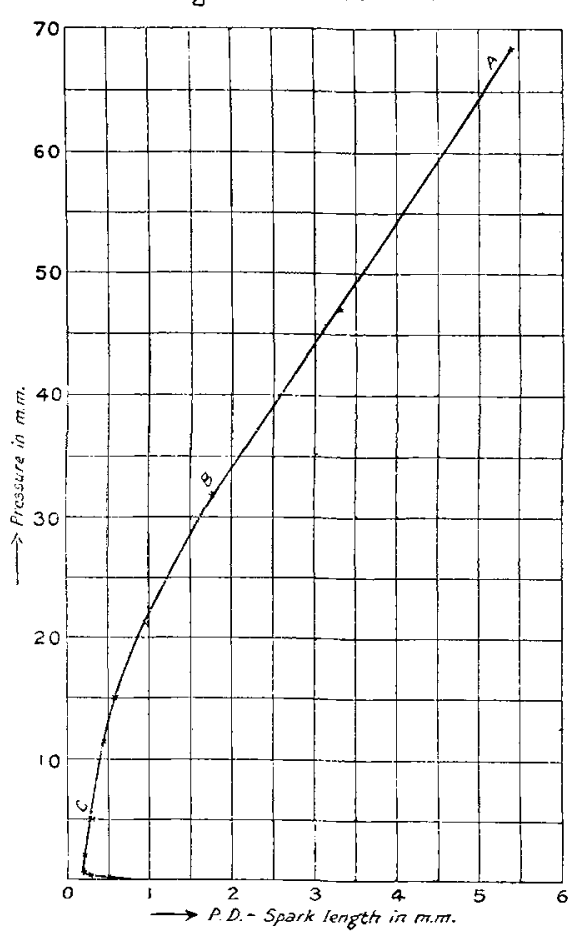

It will be seen that in curves $V$., VI, the straight portion correspending to $\mathrm{A}, \mathrm{B}$ in curve VII. is absent.

24. We conclude, therefore, that only when there is "band" discharge (which alone rotates under the influence of radial magnetic field), is the ratio of potential difference to pressure constant. This is in accordance with art. 5 .

It is further worthy of note that the potential difference goes on decreasing as pressure decreases up to a certain point; but after that, it increases with decreasing pressure, so that at very low pressures the potential difference is comparable to what it is at very high pressures.

It follows also from these experiments that the potential difference at any given pressure, although it depends on the 
voltage of the induction-coil, is materially affected by the circumstances of the discharge.

25. All these points seem to be capable of explanation on such considerations as the following :-

Let $V_{0}$ be the voltage of the induction-coil ; then the energy supplied per unit of time by the coil will be proportional to the $V_{0}$, say $i_{0} V_{0}$, where $i$ is the current in the circuit.

Let $\mathrm{V}$ be the potential difference between the electrodes; then the energy supplied to the electrodes per unit of time will be proportional to $\mathrm{V}=i^{\prime} \mathrm{V}$, say.

Therefore $i_{0} \mathrm{~V}_{0}=i^{\prime} \mathrm{V}+$ energy carried away by the positive and negative ions, thrown off from the electrodes, less the energy carried to the electrodes by positive and negative ions reaching them (per unit of time).

But the energy carried off by an ion $=\mathrm{X} e \lambda$.

Therefore,

$$
i_{0} \mathrm{~V}_{0}=i^{\prime} \mathrm{V}+\mathrm{X} e\left(\mathrm{~N} q^{\prime} \lambda^{\prime}+n q \lambda\right)-\mathrm{E},
$$

where $n$ and $\mathrm{N}$ are the numbers of positive and negative ions thrown off from the electrodes and occupying unit length of the discharge, and $\lambda, \lambda^{\prime}$ their mean free paths.

In order to find $\mathrm{E}$, we may proceed as follows :

It can be shown that the equations of continuity in a discharge-tube can be written, in the steady state,

where

$$
\left.\begin{array}{rl}
\frac{\partial \mathrm{N} q}{\partial x} & =\alpha \mathrm{N} q^{\prime}+\gamma n q, \\
\frac{-\partial(n q)}{\partial x} & =a \mathrm{~N} q^{\prime}+\gamma n q,
\end{array}\right\} . . . . .
$$

and $n, \mathrm{~N}$ the number iof positive and negative ions per unit length of discharge, $x$ being measured along the line of discharge.

Therefore, we have

$$
\mathrm{N} q^{\prime}+n q=\text { const. }={ }_{e}^{i},
$$

where $i$ is the current carried by the discharge. 
Again, the energy carried to the cathode by the positive ions may be written equal to

$$
\frac{e \mathrm{~V} x}{d} e^{-k x}\left(\alpha \mathrm{N} q^{\prime}+\gamma n q\right),
$$

where $k$ is a coefficient determining the dissipation of energy during the passage of these ions.

Also, the energy carried to the anode by the negative ions may similarly be written equal to

$$
\frac{e \mathrm{~V} a^{\prime}}{d} e^{-k^{\prime} x^{\prime}}\left(\alpha \mathrm{N} q^{\prime}+\gamma n q\right)
$$

(J. J. Thomson's ' Conduction of Electricity through Gases,' 2nd ed. p. 490),

where $d=$ distance between the electrodes;

$\mathrm{V}=$ difference of potential between the electrodes assumed to vary uniformly.

$$
\therefore \quad \mathrm{E}=\frac{e \mathrm{~V}}{d} \int\left(\alpha \mathrm{N} q^{\prime}+\gamma n q\right) x\left(e^{-k x}+e^{-k^{\prime} x^{\prime}}\right) d x .
$$

But from (5) and (6), if $\alpha, \gamma$ be regarded as constant,

$$
\begin{gathered}
\left(\alpha \mathrm{N}^{\prime} q^{\prime}+\gamma n q\right)(\alpha-\gamma)=\alpha \frac{\partial \mathrm{N} q^{\prime}}{\partial x}+\gamma \frac{\partial n q}{\partial x} ; \\
\therefore \quad \alpha \mathrm{N} q^{\prime}+\gamma n q=\left(\alpha \mathrm{N}_{1} q_{1}{ }^{\prime}+\gamma n_{1} q_{1}\right) e^{(\alpha-\gamma) x} . . . .
\end{gathered}
$$

if $\mathrm{N}=\mathrm{N}_{1}, n=n_{1}$ at the cathode.

Hence,

$$
\begin{aligned}
& \mathrm{E}=\left.\frac{\mathrm{V} e}{d}\left(\alpha \mathrm{N}_{1} q_{1}^{\prime}+\gamma n_{1} q_{1}\right)\right|_{0} ^{d^{d}} x e^{(\alpha-\gamma) x}\left(e^{-k x}+e^{-k^{\prime} x}\right) d x \\
& =\frac{\mathrm{V} e}{d}\left(\alpha \mathrm{N}_{1} q_{1}{ }^{\prime}+\gamma n_{1} q_{1}\right)\left[\frac{d e^{(\alpha-\gamma-k) d}}{\alpha-\gamma-k}-\frac{e^{(\alpha-\gamma k) d}-1}{(\alpha-\gamma-k)^{2}}+\right. \\
& =\mathrm{X} e\left(\alpha \mathrm{N}_{1} q_{1}{ }^{\prime}+\gamma n_{1} q_{1}\right) \mathrm{P} \text {, say. } \\
& \text { similar terms in } \left.k^{\prime}\right]
\end{aligned}
$$

If $\alpha=\gamma=0$, the above equation reduces to $\mathrm{E}=0$. This we may suppose to be the case during the rotatory stage in air [Phil. Mag. Oct. 1912]. Therefore, since in this case $\mathrm{N}=n$, and the pressure varies inversely as mean free path, we get the equation

$$
i_{0} \mathrm{~V}_{0}-i^{\prime} \mathrm{V}=n e \mathrm{X} \lambda^{\prime}\left(q^{\prime}+q \frac{\lambda}{\lambda^{\prime}}\right)
$$


Discharge in a Transverse Magnetic Field.

But

$$
\begin{aligned}
i_{0}=i^{\prime}+i=i^{\prime}+n e\left(q+q^{\prime}\right) ; \\
\therefore \quad \mathrm{V}_{0}-\frac{i^{\prime}}{i_{0}} \mathrm{~V}=n e \mathrm{X}^{\prime} \frac{q^{\prime}+q \frac{\lambda}{\bar{\lambda}^{\prime}}}{i^{\prime}+n e\left(q+q^{\prime}\right)}
\end{aligned}
$$

As, moreover, during this stage $V$ is small compared with $V_{0}$, and $i^{\prime}$ should be small compared with $i_{0}$, we get the simple equation

$$
\mathrm{V}_{0}=\frac{\mathrm{X}}{p}\left[\frac{q^{\prime}+q \frac{\lambda}{\lambda^{\prime}}}{q+q^{\prime}}\right] \text { or } \quad \frac{\mathrm{X}}{p}=\text { const. nearly. }
$$

This, as we have seen, is the case in air (curve VII.); when, however, the pressure is sufficiently reduced, $\alpha, \gamma$ are no longer zero. In fact, the terms in $\mathrm{H}$ become sufficiently effective in making $V$ large, as is found to be the case (art. 24), since $\alpha, \gamma, k, k^{\prime}$ are all proportional to pressure, and it is reasonable to suppose $\alpha<\gamma$, remembering that $\alpha \propto \frac{1}{\lambda^{\prime}}$ and $\gamma \propto \frac{1}{\lambda}$.

26. Although it is not possible to work out completely the theory of this variation of pressure without a knowledge of $\alpha, \gamma, k, k^{\prime}$, we may get some insight into its nature in special cases by proceeding as follows :-

From (8), we have

$$
\left(\alpha \mathrm{N}_{1} q_{1}{ }^{\prime}+\gamma n_{1} q_{1}\right) e^{(\alpha-\gamma) \delta}=\left(\alpha \mathrm{N}_{2} q_{2}{ }^{\prime}+\gamma n_{2} q_{2}\right) . .
$$

if $\mathrm{N}=\mathrm{N}_{2}^{\prime}, n=n_{2}$ at the anode, $q=q_{2}$ and $q=q^{\prime} / q_{2}^{\prime}$;

but

$$
e\left(\mathrm{~N}_{2} q_{2}{ }^{\prime}+n_{2} q_{2}\right)=i=e\left(\mathrm{~N}_{1} q_{1}{ }^{\prime}+n_{1} q_{1}\right) . \quad .
$$

If, now, $n_{2}=0$,

We have also

$$
\frac{\alpha i}{\alpha-\gamma} e^{(\nu-\alpha) d}-\frac{\gamma^{i}}{\alpha-\gamma}=e \mathrm{~N}_{1} q_{1}{ }^{\prime}
$$

$$
i_{0} \mathrm{~V}_{0}-i^{\prime} \mathrm{V}=\mathrm{X}_{e} \mathrm{~N}_{1} q_{i}^{\prime}\left[\lambda^{\prime}-\alpha \mathrm{P}\right] \text {, since } n_{2}=0 ;
$$

and since

$$
i^{\prime}=\text { small, }
$$

we get $\mathrm{X}$ as an exponential function of $p$.

It is obvious, however, that the above investigation is not capable of giving a complete account of the variation of the potential difference, for we have assumed (1) that the potential varies uniformly from cathode to anode, and (2) that $\alpha, \gamma$ are constant. As neither of these suppositions can be true always, it is not surprising that the curves obtained are more complicated than those given by theory. 
64 Electric Discharge in a Transverse Magnetic Field.

27. It is not without interest to follow the variation of current with pressure and potential difference.

Curves VIII., IX., fig. 22, give the relation between current and pressure in a discharge-tube (No. 1), the induction-coil used giving a spark-length, in air, of 13*4.

Fig. 22. Curves VIII. \& IX.

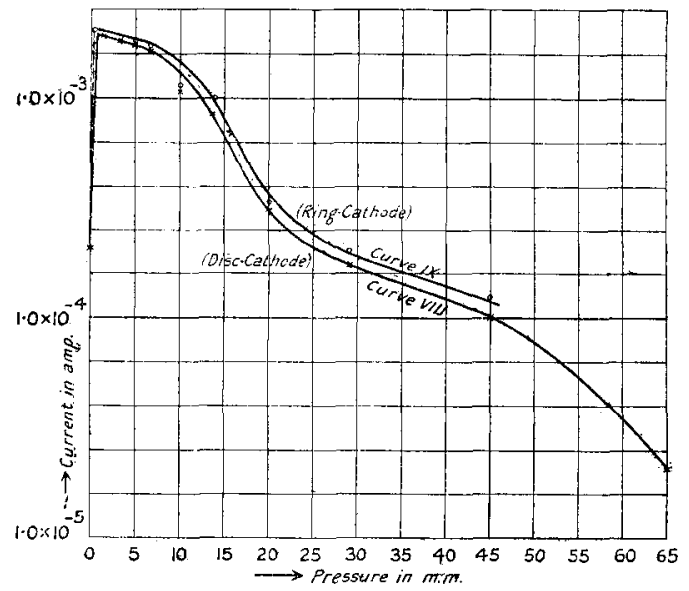

Fig 23 Curve. X.

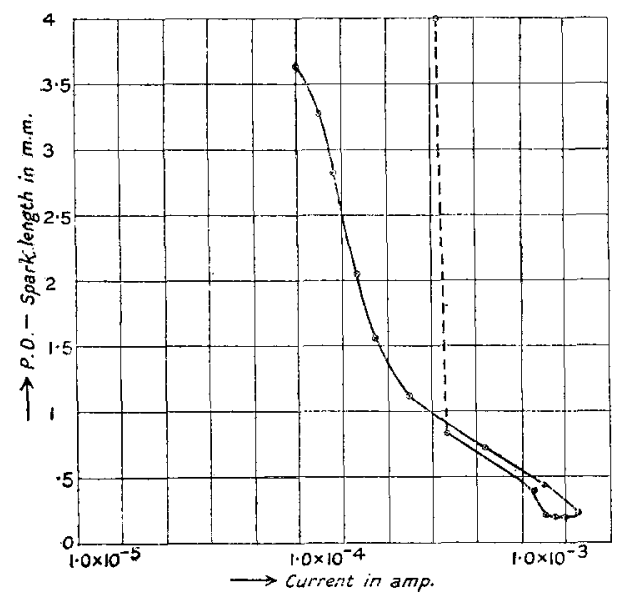

The curve X., fig. 23, gives the relation between the current and potential difference. 
From (9), (10) we have

We have also

$$
e n_{2} q_{2}=\frac{\alpha i}{\alpha-\gamma}-\left(e \mathrm{~N}_{1} q_{1}^{\prime}+\frac{\gamma i}{\alpha-\gamma}\right) e^{(\alpha-\gamma) d} .
$$

$$
i \mathrm{~V}_{0}=\mathrm{X} e\left(\mathrm{~N}_{1} q_{1}{ }^{\prime} \lambda^{\prime}+n_{2} q_{2} \lambda\right)-\mathbf{E} ;
$$

so that a third relation is required in order that we should know $\mathrm{N}_{1}, n_{2}$, and $i$.

According to J. J. Thomson's theory, when there is only one kind of ion in the tube,

$$
\sqrt{ } i=a \mathrm{~V}+b \text {. }
$$

It is our intention to discuss the experimental results in their bearing on this and other theories in a future paper.

Our thanks are due to the authorities of the Presidency College, Calcutta, for affording us facilities for carrying the above investigations.

\section{Results of Crystal Analysis.}

By L. Vegard, Dr. phil., University of Christiania* .

\section{[Plate III.]}

$\S 1$. TN a previous paper $\uparrow \mathrm{I}$ gave an account of the 1 crystalline structure of silver, as determined by the Bragg reflexion method. The $\mathrm{X}$-ray spectrometer was in principle the same as that constructed by Bragg, only differing with regard to details which were mentioned in the paper.

Since then the work has been continued, and some of the results obtained will be given in this paper.

Besides some results concerning the structure of gold and lead, which were announced in a paper read before "Kristiania Videnskapselskap" on November 19, 1915, the present paper will chiefly deal with the more elaborate and complicated case, the determination of the structure of the Zircon group, represented by the minerals zircon $\left(\mathrm{ZrSiO}_{4}\right)$, rutile $\left(\mathrm{TiO}_{2}\right)_{2}$, and tinstone (kassiterite) $\left(\mathrm{SnO}_{2}\right)_{2}$, which are, as far as I know, the first cases of tetragonal crystals which have yet been analysed $\ddagger$.

Not being aware of the fact that the Spinel group recently has been analysed by W. H. Bragg $\S, I$ have also made an

* Communicated by the Author.

$\dagger$ L. Vegard, Phil. Mag. Jan. 1916.

I An account of the analysis of the Zircon group was giren in a lecture in Kristiania Vid. Selsk. March 10, 1916.

$\S$ W. H. Bragg, Phil. Mag. Aug. 1915, p. 305.

Phil. Mag. S. 6. Vol. 32. No. 187. July 1916. 


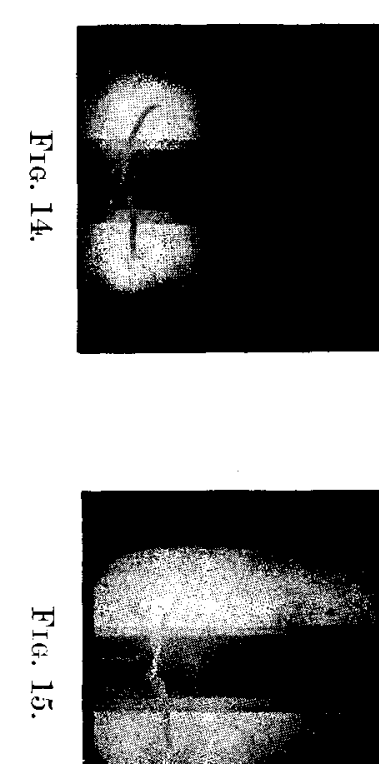

1

11
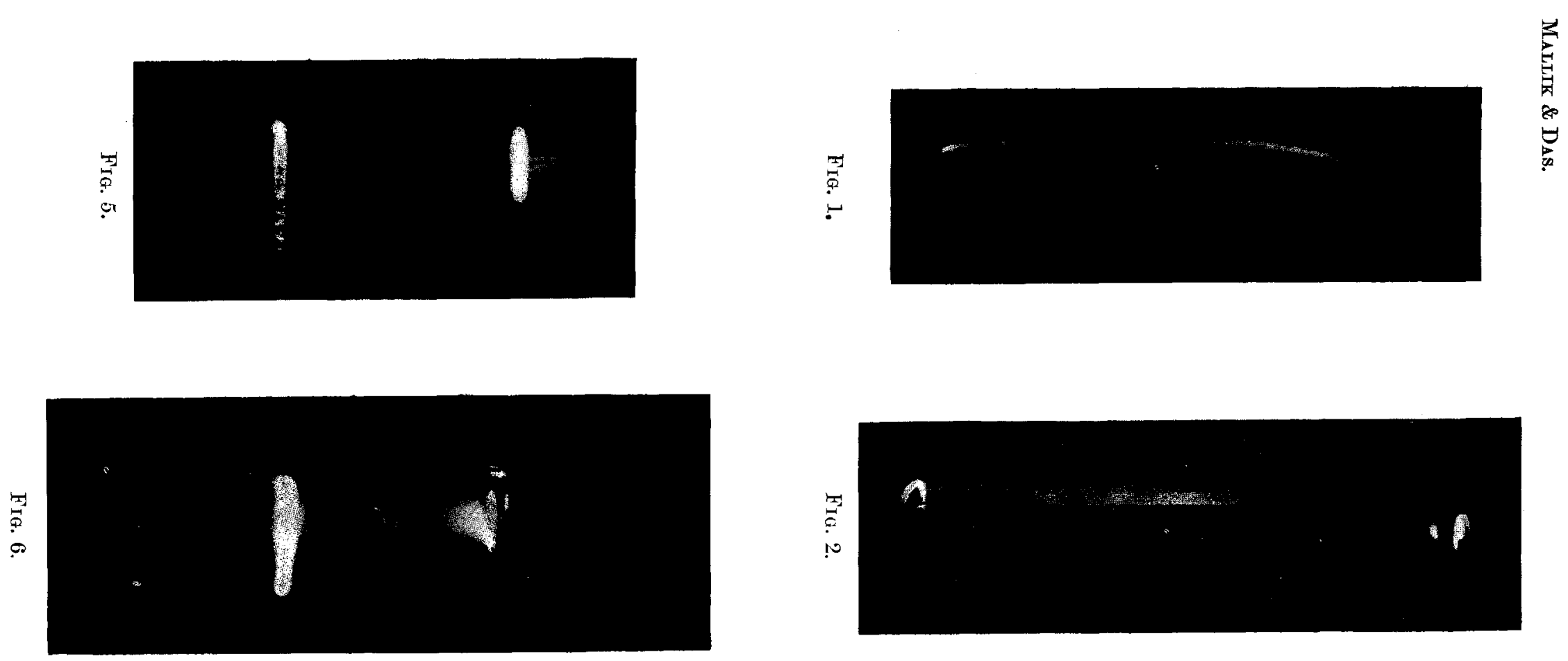

16r.

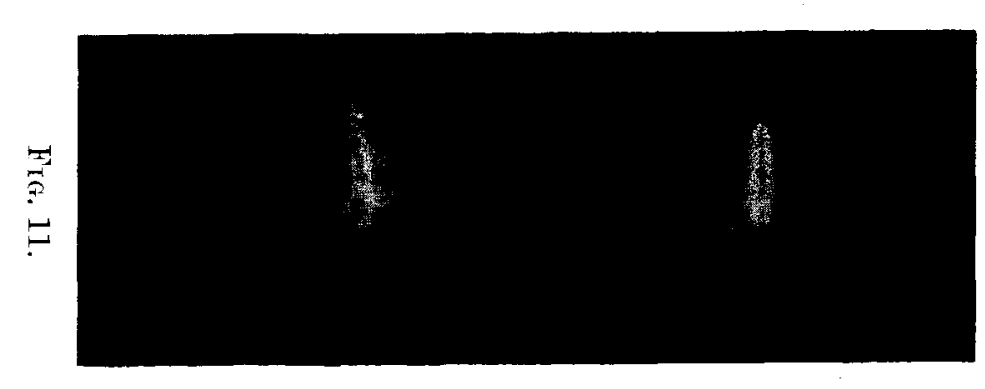

$+1$

$E-x_{0}=0$

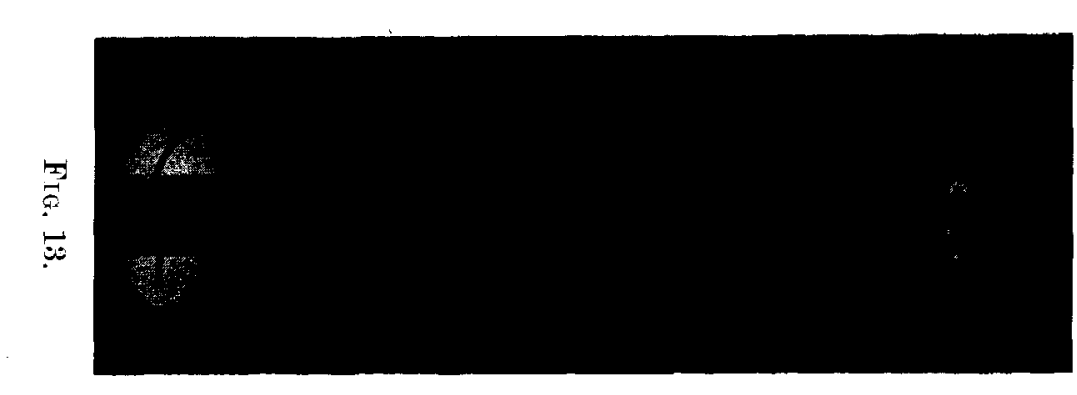

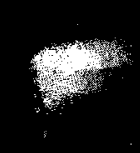

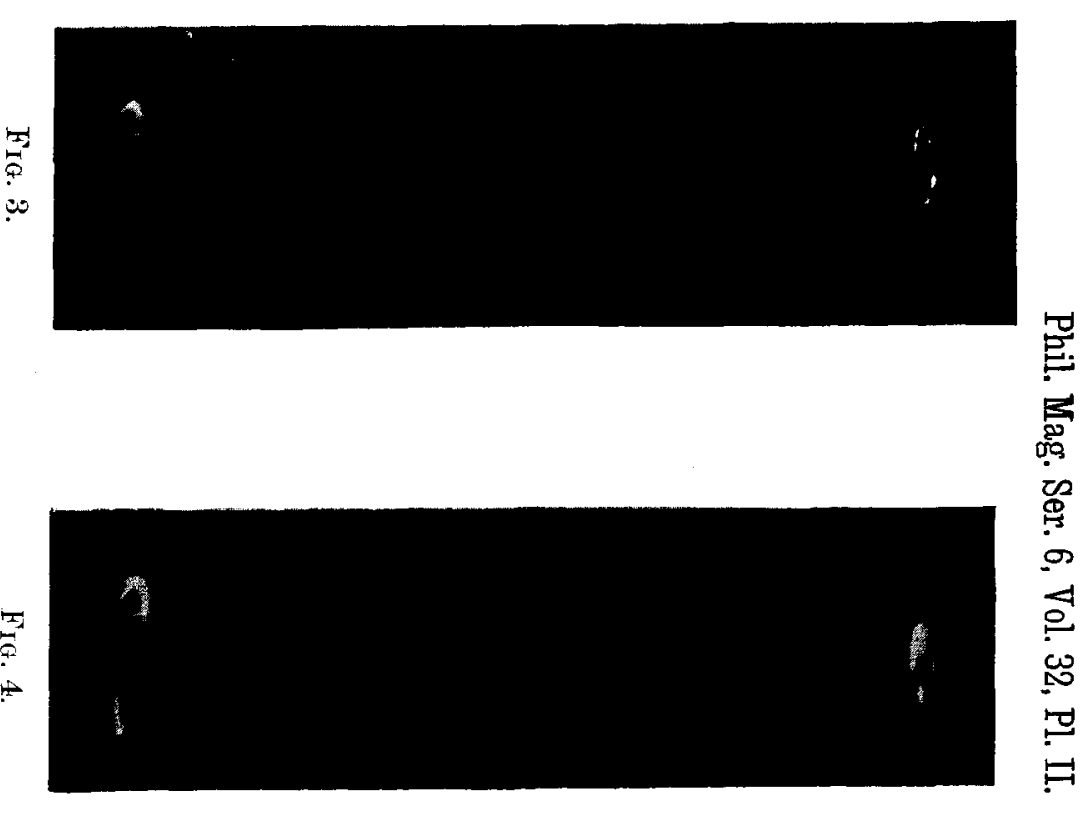

